\title{
Primary frequency response based rescheduling for enhancing microgrid resilience
}

\author{
Liang CHE $^{1}$ (D) Xinwei SHEN ${ }^{2}$, Mohammad SHAHIDEHPOUR ${ }^{3}$
}

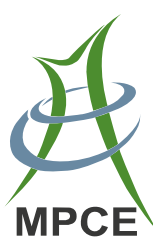

\begin{abstract}
The existing microgrid operation schemes do not consider the dynamic performance of frequency in the islanded operation of microgrids. When an islanded microgrid encounters a disturbance, the sudden power mismatch could impose security risks or even a system collapse. To address such a challenge, this paper proposes the primary frequency response rescheduling (PFRR) approach. For a certain operation interval, the PFRR will optimally reschedule the distributed generators (DGs) with non-zero mechanical inertia and adjust the battery power. And the objective is to limit the rate-of-change-of-frequency (ROCOF) and to maintain the post-disturbance frequency nadir above a prescribed threshold. The effectiveness of the proposed strategy is verified by a case study on the IEEE 123-node test feeder system and the timedomain simulation in MATLAB Simulink.
\end{abstract}

Keywords Microgrid, Inertia, Frequency control, Primary frequency response, Resilience

CrossCheck date: 28 May 2019

Received: 19 July 2018/ Accepted: 28 May 2019/Published online: 9 July 2019

(C) The Author(s) 2019

$\square$ Liang CHE

lche@hawk.iit.edu

Xinwei SHEN

sxw.tbsi@sz.tsinghua.edu.cn

Mohammad SHAHIDEHPOUR

ms@iit.edu

1 Hunan University, Changsha 410082, Hunan, China

2 Tsinghua-Berkeley Shenzhen Institute, Shenzhen 518055, China

3 Illinois Institute of Technology, Chicago, IL 60616, USA

\section{Introduction}

The increasing frequency of extreme events and the operational uncertainties underscore the importance of enhancing the resilience, i.e., the ability of a system to prepare for and adapt to changing conditions, and withstand and recover rapidly from extreme outages [1-3]. The deployment of microgrids, which are self-controlled entities with the capability to operate in islanded mode, is an important way of enhancing the resilience of distribution systems. An islanded microgrid can encounter disturbances, e.g., the unintentional islanding, the outage of generation resource, and large changes of load. Such disturbances will cause an instantaneous power imbalance. Due to the well-known low-inertia property of microgrids, such imbalance can immediately cause a rapid deviation of frequency, and thus challenge the operation of microgrid or lead to instabilities. This is one of the most challenging issues for islanded microgrid operations. In case of low frequency, the microgrid will be vulnerable to additional disturbances on such occasions as the key microgrid parameters are designed based on the rated operation states. If the frequency falls below its set point for a while, some distributed generation resources (DERs) could stop working, resulting in additional power deficit. Even though the frequency collapse may be averted using load shedding schemes, the large frequency deviation may pose a threat to microgrid stability.

Microgrids involve the large-scale deployment of lowinertia DERs which are typically inverter-coupled PVs and energy storage units, and wind turbines. This has led to a high share of low-inertia resources and has greatly reduced rotational inertia of microgrids. Consequently, the loss of rotational inertia leads to new frequency instability phenomena in microgrids. This is notably a concern for both 
the steady-state operation of microgrid and the system resilience after disturbances. A low-inertia level will render the frequency stabilization and restoration after a disturbance more difficult. If the frequency cannot be stabilized and restored while satisfying the performance criteria, e.g., the limits on rate-of-change-of-frequency (ROCOF) and frequency nadir, then the frequency deviation can cause vibrations in machines, damage critical facilities or lead to transient instabilities. This underscores the need for appropriate enhancement of frequency stabilization methods to enhance microgrid resilience.

The microgrid inertia problem may not be fully addressed by the recently proposed virtual inertia (VI) techniques [4-6]. Firstly, the VI techniques typically provide the VI by regulating the DERs' response. This paper investigates the low inertia issue which is the adequacy of the inherent rotational inertia of the system. Secondly, the VI is not an inherent physical behavior, but relies on detecting frequency deviation or ROCOF based on the power electronic control loops. Such control loops use "the active power-frequency $(P-\omega)$ control" and operate "converters as virtual synchronous machines" to "emulate inertia, damping characteristic and the primary frequency regulation" [4], vary the DER power "according to the grid frequency" [5], "reduce frequency deviations in microgrids by modifying inverter control" based on the detection of frequency [6].Thus, the VI techniques basically adjust the response of DERs after detecting the frequency deviations. Hence, supposing a large disturbance occurs at $t=0$, the VI will be delivered after a time delay $\Delta t$, which is the total time needed for implementing all of the following processes: the detection of frequency change, data transmission, signal processing, computation, converter control, and the process of increasing the DER's power output by the converter control. However, the ROCOF equals to the instantaneous power imbalance divided by the inertia. Therefore, when a low-inertia microgrid encounters the aforementioned disturbance, it is likely that the large imbalance and lower-inertia will cause a large magnitude of ROCOF and thus lead to large frequency deviation within $\Delta t$, i.e., before the delivery of VI. Therefore, the VI based approaches may not fully address the low inertia issue of islanded microgrids especially in the case of large disturbance.

When an islanded microgrid encounters a large power imbalance after a disturbance, the load shedding schemes, e.g., the under-frequency load shedding (UFLS) [7-10], are used to prevent the system collapse. In such load shedding schemes, it is a challenging task to determine the load curtailment amount at each step by estimating the power deficiency [7]. The recently proposed adaptive load shedding schemes [8, 9] estimate power imbalances by measuring frequency derivatives, determining the optimal amount of load to be shed, or implementing multi-step load shedding, which can help avoid the excessive curtailment. By estimating the ROCOF in the equivalent center of inertia, adaptive load shedding at each step corresponding to the frequency control response can be devised. However, the low inertia issue of microgrids will challenge the load shedding schedule as it requires the load shedding schemes response to the disturbance faster and more accurate.

To undertake this challenge, this paper proposes the primary frequency response rescheduling (PFRR) approach. For a certain operational interval, the PFRR will optimally reschedule the distributed generators (DGs) that have non-zero mechanical inertia and adjust the battery power, with the objective to limit the ROCOF and maintain the frequency nadir after the disturbance above a prescribed threshold. The contributions of the paper are listed as follows.

1) The PFRR strategy is proposed for enhancing the microgrid resilience in terms of frequency dynamics when the islanded microgrid encounters disturbances. The PFRR can ensure the ROCOF and frequency nadir performance criteria.

2) The relationships among system inertia, ROCOF, frequency nadir, primary frequency response (PFR) reserve and the microgrid primary/secondary controls are analyzed and investigated.

3) This work contributes to further investigation of effective resolution to the low inertia issue of microgrids.

The remainder of this paper is organized as follows. Section 2 introduces the background information. Section 3 presents the proposed PFRR, which is validated by detailed simulations in Section 4. Finally, Section 5 concludes the paper.

\section{Background}

The inertia, PFR, and how the PFR in the bulk power systems is modeled in the literature, are discussed as follows.

\subsection{Inertia}

The kinetic energy stored in a rotational mass is proportional to its moment of inertia and the square of angular velocity. For a synchronous generator, the angular speed is directly coupled with the system frequency. The amount of the kinetic energy in the system and thus the system inertia is determined by the rational inertia of the online rotating machines. In case of a disturbance mentioned in Section 1, a sudden power imbalance occurs. In the case of a power 
deficit caused by a loss of generation (due to either unintentional islanding or generation outage), the power deficit is automatically and instantaneously balanced by the inherent physical behavior of the rotating machines which draw on their stored mechanical kinetic energy to fill the gap between the generation and load. This is completed instantaneously without any time delays because the electrical powers (generation and consumption) in the system are balanced all the time and the frequency (coupled with the angular speed of synchronous generators) will immediately decrease after the disturbance. Hence, the conversion of the kinetic energy to the electrical energy will result in a slow-down of the synchronous generators and a drop in frequency. The inertia constants of conventional synchronous generators are in the order of 2-9 s [11]. The inertia can be provided by both generators and motors, although we focus on the generation in this work.

The analysis is analogous for the power surplus case (e.g., caused by microgrid islanding or loss of load).

\subsection{PFR}

Microgrids are operated in the primary, secondary and tertiary control levels (tertiary is the highest level), which provide the functions of power control, frequency/voltage restoration and system-wide coordination, respectively. A higher control level has a longer time scale. The PFR includes the primary control and the system frequency dynamics in response to a sudden power mismatch, where the latter is determined by the inertia. The traditional concept of microgrid "primary control" does not involve the PFR and frequency dynamics as it implicitly assumes that the system has adequate inertia.

The system frequency dynamics are determined by the swing equation:

$\frac{\mathrm{d} f(t)}{\mathrm{d} t}=\frac{1}{H}\left(P_{m}-P_{e}\right)$

where $\frac{\mathrm{d} f(t)}{\mathrm{d} t}$ is the ROCOF; $H$ is the inertia; and $f$ is the frequency. In normal operations, the power mismatch $P_{m}-$ $P_{e}$ is small, and $f(t)$ is close to the rated frequency. In the power deficit case, $P_{m}$ reduces and the value of power mismatch becomes significant (negative), and the frequency starts declining as determined by (1). Next, the microgrid primary control begins to take action. For synchronous gentrater couple DERs, their governors will respond by increasing mechanical power to re-establish the balance between $P_{m}$ and $P_{e}$. For inverter-coupled DERs, their primary control loop will increase the power output according to the sensed frequency drop as determined by their droop characteristics. The system frequency does not show significant variations across buses even in a largescale power system [11]. So for a microgrid, it is reasonable to assume that the system frequency is the same at different nodes in the microgrid.

The limitation of the VI techniques lies in the fact that a microgrid with a higher share of inverter-coupled DERs has a lower value of $H$. Therefore, according to (1), it will have very large ROCOF when encountering a power deficit. Such a large ROCOF is likely to cause a very large frequency deviation within $\Delta t$, i.e., a very large value of $\frac{\mathrm{d} f(t)}{\mathrm{d} t} \Delta t$. Here $\Delta t$ is used to denote the time delay of delivering the VI, i.e., the time between the starting of the disturbance (at which the frequency starts changing) and the full delivery of the VI. As mentioned earlier, it includes the time needed for implementing the detection of frequency change, data transmission, signal processing, computation, converter control, and the process of increasing the DER's power output by the converter control. In contrast, the rotational inertia is built on the inherent physical law and always supports the frequency instantaneously.

\subsection{Modeling PFR and governor ramp}

The PFR problem is first analyzed in [12], which developes a detailed model of the governor primary response for generation dispatch. Later, governor rateconstrained optimal power flow and dynamics-constrained frequency unit commitment are examined in $[13,14]$.

Governor ramp rate $c_{g}$ represents the prime mover ramping restriction, i.e., the fastest rate of change of mechanical power output in response to sudden frequency deviations [15]. Reference [15] proves that the necessary (but insufficient) condition for ensuring the frequency nadir limit is:

$\left\{\begin{array}{l}R_{g} \leq P_{g}^{\text {nad }}=\frac{2 H\left(f_{0}-f_{\text {nad }}\right)}{L} c_{g} \\ \sum_{g} R_{g} \geq L\end{array}\right.$

where $f_{0}$ and $f_{\text {nad }}$ are the nominal frequency and nadir threshold, respectively; $R_{g}$ is the PFR reserve of unit $g$; $p_{g}^{\text {nad }}$ is the upper limit of $R_{g}$; and $L$ is the power mismatch imposed by the disturbance. Equation (2) means that when a system with the inertia of $H$ and individual governor ramp rate of $c_{g}$ encounters a sudden power loss of size $L$, its frequency can be kept above the nadir $f_{\text {nad }}$ if its total governor primary response reserve $\sum_{g} R_{g}$, constrained in (2), is enough to cover $L$. 


\section{The proposed PFRR approach}

\subsection{Frequency nadir criterion}

Different from [15, 16], the DER with non-zero mechanical inertia in a microgrid may or may not have a governor in its prime mover. However, the PFR mechanism discussed in [15] still applies to this paper. Therefore, we use a more general term source ("source power" and "source ramp rate" which replaces "mechanical power" and "governor ramp rate" in [15], respectively) when discussing the physical ramping restrictions in the prime mover or power source of a controllable DER in the microgrid.

The development of PFR constraint is presented below. In case of a disturbance (due to generation outage, load step change, unintentional islanding), the microgrid experiences a sudden power shortage of magnitude $L$. The dynamic response of the overall source power to the mismatch with a constant overall source ramp rate of $c$ is depicted in Fig. 1 , where $\bar{P}^{d i s}$ and $P^{\text {batt }}$ are the maximum discharging power and the power of the energy storage; $\mathrm{t}_{B}$ is the time delay of dead band [13]; and $c$ denotes a constant overall source ramp rate of $c$. In $[15,16]$, the source power and the source ramp rate are the mechanical power and its ramp rate of generators in the bulk power system, respectively. In this paper, they refer to the mechanical or source power input of a controllable DG and its ramp rate (the DG may or may not be converter-coupled), respectively. The ramp rate is not constant in reality, but a constant rate is used here and it is assumed that such constant value is a conservative estimate so that the response will be always faster than estimated.

The derivation of the constraints of the proposed PFRR is given below. For the PFR model depicted in Fig. 1, integrating (1), we have:

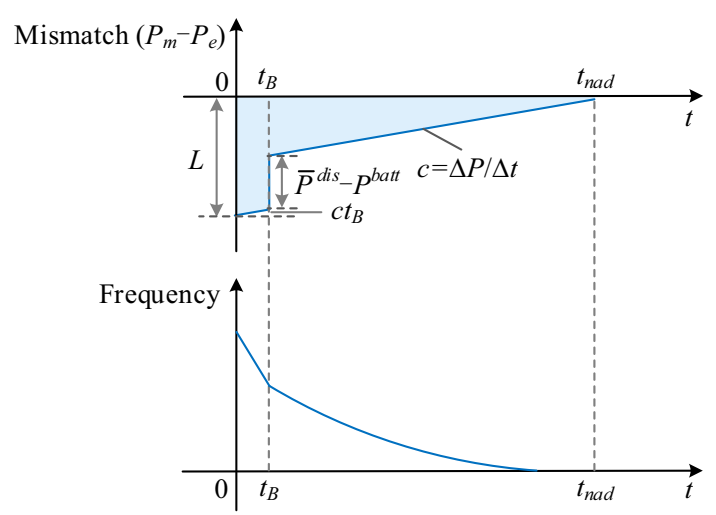

Fig. 1 PFR model

$$
\begin{aligned}
\int_{0}^{t_{\text {nad }}} \frac{\mathrm{d} f(t)}{\mathrm{d} t} \mathrm{~d} t & =f\left(t_{\text {nad }}\right)-f(0)=f_{\text {nad }}-f_{0} \\
& =\frac{1}{H} \int_{0}^{t_{\text {nad }}}\left(P_{m}(t)-P_{e}(t)\right) \mathrm{d} t
\end{aligned}
$$

Therefore,

$H\left(f_{0}-f_{\text {nad }}\right)=\int_{0}^{t_{\text {nad }}}\left(P_{e}(t)-P_{m}(t)\right) \mathrm{d} t$

The right-hand side of (4) is geometrically represented by the area of the shaded region in Fig. 1. Hence, (4) is transformed to:

$$
\begin{aligned}
H\left(f_{0}-f_{\text {nad }}\right)= & \left(L-\frac{1}{2} c t_{B}\right) t_{B} \\
& +\frac{\left(L-c t_{B}-\sum_{j}\left(\bar{P}_{j}^{d i s}-P_{j}^{\text {batt }}\right)\right)^{2}}{2 c}
\end{aligned}
$$

where $\bar{P}_{j}^{d i s}$ is maximum discharging power of battery $j$; and $P_{j}^{\text {batt }}$ is the power of the energy storage unit $j$ (its positive and negative values denote the discharging and charging powers, respectively). Considering that $c t_{B}<<L$, we ignore $c t_{B}$ on the right-hand of (5). According to [15], we can obtain the minimal overall ramp rate from (5):

$c_{\text {min }}^{U P}=\frac{1}{2} \frac{\left(L-\sum_{j}\left(\bar{P}_{j}^{\text {dis }}-P_{j}^{\text {batt }}\right)\right)^{2}}{H\left(f_{0}-f_{\text {nad }}\right)-L t_{B}}$

Next, we derive the frequency nadir constraint as follows. According to [15], the PFR constraint for a single unit $i$ is:

$R_{i}^{U P} \leq c_{i} \Delta t=c_{i} \frac{L-\sum_{j}\left(\bar{P}_{j}^{d i s}-P_{j}^{\text {batt }}\right)}{c_{\text {min }}^{U P}}$

where $R_{i}^{U P}$ and $c_{i}$ are the PFR reserve and the PFR governor ramp rate of non-zero-inertia DGs (NZI-DG) $i$, respectively. Substitute (6) into (7), we have:

$R_{i}^{U P} \leq 2 c_{i} \frac{H\left(f_{0}-f_{\text {nad }}\right)-L t_{B}}{L-\sum_{j}\left(\bar{P}_{j}^{d i s}-P_{j}^{\text {batt }}\right)}$

Therefore, (8) together with (9) are sufficient conditions for the system to satisfy the PFR criterion in case of power shortage:

$\sum_{i} R_{i}^{U P} \geq L-\sum_{j}\left(\bar{P}_{j}^{d i s}-P_{j}^{\text {batt }}\right)$

In case of a sudden power surplus of magnitude $L$ caused by a disturbance, analogous derivation can be performed and therefore (10) together with (11) are sufficient conditions for the system to satisfy the PFR criterion: 
$R_{i}^{D N} \leq 2 c_{i} \frac{H\left(f_{\text {nad }}-f_{0}\right)-L t_{B}}{L-\sum_{j}\left(\bar{P}_{j}^{c h}+P_{j}^{b a t t}\right)}$

$\sum_{i} R_{i}^{D N} \geq L-\sum_{j}\left(\bar{P}_{j}^{c h}+P_{j}^{b a t t}\right)$

where $\bar{P}_{j}^{c h}$ is charging power (positive parameter) of battery $j$; and $R_{i}^{D N}$ is the PFR reserve when DG $i$ is ramping down.

Particularly, in the case of resource $i$ being limited by its generation capacity (i.e., its capacity reserve is less than the right-hand side of (8) or (10)), its generation capacity constraint will ensure $R_{i}$ can be delivered before $t_{\text {nad }}$. Otherwise, constraint (8) or (10) will be active and will ensure $R_{i}$ can be delivered before $t_{\text {nad }}$. Furthermore, $c_{i}$ is used in both (8) and (10) as we assume the same ramp rate in up and down directions. If they are not the same, different parameters should be used.

$c_{i}$ can be obtained from stress test (a measurement of the generation resources) [17] and $H$ can be estimated from empirical data [18] or online testing [19].

\subsection{ROCOF criterion}

ROCOF is limited by the constraint below:

$\gamma_{0}=\frac{\mathrm{d} f(t)}{\mathrm{d} t}=\frac{L}{2 H} \leq \gamma_{0}^{\max }$

$\gamma^{U P}=\frac{\mathrm{d} f(t)}{\mathrm{d} t}=\frac{L-\sum_{j}\left(\bar{P}_{j}^{\text {dis }}-P_{j}^{\text {batt }}\right)}{2 H} \leq \gamma_{\max }^{U P}$

$\gamma^{D N}=\frac{\mathrm{d} f(t)}{\mathrm{d} t}=\frac{L-\sum_{j}\left(\bar{P}_{j}^{c h}+P_{j}^{\text {batt }}\right)}{2 H} \leq \gamma_{\max }^{D N}$

where $\gamma$ is the ROCOF; $\gamma_{0}$ is immediately after the disturbance $\left(t=0^{+}\right)$; and $\gamma^{U P}$ and $\gamma^{D N}$ are the ROCOF after immediately battery discharging and charging in powershortage and power-surplus cases, respectively. $\gamma_{0}$ is considered because battery storage or any VI device cannot provide mechanical/rotational inertia as discussed in Section 2. Immediately after the disturbance $\left(t=0^{+}\right)$, ROCOF of the system is arrested by $H$. Hence, the ROCOF constraint is given as (12). In fact, constraint (12) not only limits the ROCOF caused by the disturbance but also indicates a fact that the microgrid should have enough mechanical/rotational inertia to support its frequency dynamic response immediately after the disturbance.

The system inertia is calculated as:
$H=\frac{1}{f_{0}} \sum_{i} J_{i} P_{i}^{\max }$

where $J_{i}$ is the inertia constant of unit $I ; f_{0}$ is the nominal frequency; and $H$ is in $(\mathrm{kW} \cdot \mathrm{s} / \mathrm{Hz})$. Hence, substitute (15) into (12)-(14), the ROCOF constraints are written as:

$\sum_{i} J_{i} P_{i}^{\max } \geq \frac{L f_{0}}{2 \gamma_{0}^{\max }}$

$\sum_{i} J_{i} P_{i}^{\max } \geq \frac{\left(L-\sum_{j}\left(\bar{P}_{j}^{\text {dis }}-P_{j}^{\text {batt }}\right)\right) f_{0}}{2 \gamma_{\max }^{U P}}$

$\sum_{i} J_{i} P_{i}^{\max } \geq \frac{\left(L-\sum_{j}\left(\bar{P}_{j}^{c h}+P_{j}^{b a t t}\right)\right) f_{0}}{2 \gamma_{\max }^{D N}}$

where the different ROCOF limit can be specified.

\subsection{PFRR model}

In this paper, the DGs with non-zero mechanical inertia (typically micro-turbines) are referred to as NZI-DGs. While in comparison, the converter-coupled wind and PV units which provide very small inertia are referred to as zero-inertia DGs (ZI-DGs).

The goal of the proposed PFRR is to reschedule the NZI-DG and/or adjust the battery power to provide inertia and PFR ramp capability for the purpose of enhancing the dynamic performance of microgrid frequency in the given operational interval.

The PFRR is formulated below:

$\min \boldsymbol{s}^{\mathrm{T}} \boldsymbol{I}+\boldsymbol{\alpha}^{\mathrm{T}} \boldsymbol{P}+\boldsymbol{\beta}^{\mathrm{T}} \boldsymbol{B}$

s.t.

$I_{i} \underline{P}_{i} \leq P_{i} \leq I_{i} \bar{P}_{i}$

$\left\{\begin{array}{l}P_{i}+R_{i}^{U P} \leq I_{i} \bar{P}_{i} \\ P_{i}-R_{i}^{D N} \geq I_{i} \underline{P}_{i}\end{array}\right.$

$\left\{\begin{array}{l}\mathbf{0} \leq \boldsymbol{R}^{U P} \leq \overline{\boldsymbol{R}^{U P}} \\ \mathbf{0} \leq \boldsymbol{R}^{D N} \leq \overline{\boldsymbol{R}^{D N}}\end{array}\right.$

$-\overline{\boldsymbol{P}}^{c h} \leq \boldsymbol{P}^{b a t t} \leq \overline{\boldsymbol{P}}^{\text {dis }}$

$\left\{\begin{array}{l}\boldsymbol{B} \geq \boldsymbol{P}^{\text {batt }} \\ \boldsymbol{B} \geq-\boldsymbol{P}^{\text {batt }}\end{array}\right.$ 


$$
\begin{aligned}
& \left\{\begin{array}{l}
R_{i}^{U P} \leq 2 c_{i} \frac{H\left(f_{0}-f_{\text {nad }}\right)-L t_{B}}{L-\sum_{j}\left(\bar{P}_{j}^{d i s}-P_{j}^{\text {batt }}\right)} \\
R_{i}^{D N} \leq 2 c_{i} \frac{H\left(f_{\text {nad }}-f_{0}\right)-L t_{B}}{L-\sum_{j}\left(\bar{P}_{j}^{c h}+P_{j}^{\text {batt }}\right)}
\end{array}\right. \\
& \left\{\begin{array}{l}
\sum_{i} R_{i}^{U P} \geq L-\sum_{j}\left(\bar{P}_{j}^{\text {dis }}-P_{j}^{\text {batt }}\right) \\
\sum_{i} R_{i}^{D N} \geq L-\sum_{j}\left(\bar{P}_{j}^{c h}+P_{j}^{\text {batt }}\right)
\end{array}\right. \\
& \left\{\begin{array}{l}
\sum_{i} I_{i} J_{i} P_{i}^{\max } \geq \frac{L f_{0}}{2 \gamma_{0}^{\max }} \\
\sum_{i} I_{i} J_{i} P_{i}^{\max } \geq \frac{\left(L-\sum_{j}\left(\bar{P}_{j}^{\text {dis }}-P_{j}^{\text {batt }}\right)\right) f_{0}}{2 \gamma_{\max }^{U P}}
\end{array}\right. \\
& \sum_{i} I_{i} J_{i} P_{i}^{\max } \geq \frac{\left(L-\sum_{j}\left(\bar{P}_{j}^{\text {ch }}+P_{j}^{\text {batt }}\right)\right) f_{0}}{2 \gamma_{\max }^{D N}}
\end{aligned}
$$

In the model, $\boldsymbol{P}$ and $\boldsymbol{P}^{\text {batt }}$ are the vectors of NZI-DG power and battery power, respectively; $\bar{P}_{i}$ and $\underline{P}_{i}$ are the upper and lower power limits of unit $i$; and $J_{i}$ is the inertia time constant of unit $i$. In (19), $\boldsymbol{\alpha}, \boldsymbol{\beta}$ and $\boldsymbol{s}$ are the cost factors; $\boldsymbol{B}$ is the vector for calculating battery operational cost; and the three terms are the start-up cost, DG operational cost, and battery charging/discharging cost (accounting for battery degradation), respectively. Binary variable $I_{i}$ is the NZI-DG status indicator $\left(I_{i}=1 / 0\right.$ mean $\mathrm{ON} / \mathrm{OFF}$ ).

Constraints (20)-(22) place the bounds on the DG power $P_{i}$ and PFR reserves $R_{i}^{U P}$ and $R_{i}^{D N}$. Constraints (23)-(24) are the battery power constraints. Constraints (25)-(27) are the PFR constraints proposed earlier. It is noticed that (25), after being reformulated, will include the bilinear terms $R_{i}^{U P} P_{j}^{b a t t}$ and $R_{i}^{D N} P_{j}^{b a t t}$. They are linearized using McCormick's envelope [20].

\section{Case Study}

\subsection{Simulation setup}

In this section, the proposed PFRR is validated by simulations on the modified IEEE 123-node test feeder system. Details about the system can be found in [3] and [21]. The optimization problems are solved by CPLEX 12.4 and the time-domain simulation is performed in MATLAB Simulink.

The test system forms an islanded microgrid. Ten NZIDGs are connected in the system (the connection locations are depicted in [3]). Each of them has a capacity of $\bar{P}_{i}=$ $100 \mathrm{~kW}, \underline{P}_{i}=0$, governor (PFR) ramp rate of $c_{i}=30 \mathrm{~kW} / \mathrm{s}$, and inertia time constant of $J_{i}=3 \mathrm{~s}$. The battery has the largest charging/discharging power of $100 \mathrm{~kW}$ and action time assumed as $t_{B}=0.001 \mathrm{~s}$ (the battery parameter). In each case, the base-case load is $500 \mathrm{~kW}$, the power change (surplur/shortage) is applied by a step change of load. It is assumed that the collected mechanic inertia provided by all the ZI-DGs is equal to the inertia of a single NZI-DG.

The simulation is performed using the modified governor (DEGOV) model [22] integrated with microgrid primary and secondary control functions depicted in Fig. 2, where $\omega, \omega_{0}, \omega_{2 n d}$ and $\omega^{\text {ref }}$ denote the detected, rated, second-control and reference frequencies, respectively, and a symbol $T$ with a subscript is a time constant in the control.The default DEGOV parameters used in the industry are provided in [22]. Considering that microgrids can have smaller time constants, the time constants (parameters) are modified as $T_{1}=1 \mathrm{~s}, T_{2}=T_{5}=T_{6}=0.2 \mathrm{~s}, T_{3}=T_{4}=1 \mathrm{~s}, T_{d}$ $=0.01 \mathrm{~s}, K=1, T_{\max } / T_{\min }=1 / 0$ p.u.. The detailed microgrid primary and secondary control functions are given in [2]. $c_{i}$ can be estimated from the governor stress test [17]. In dynamic simulations, $c_{i}$ which represents the fastest ramp rate of governor mechanical power is implemented in the governor model by adding a rating limiter to the output as proved in [16]. In Fig. 2, a rate limiter $\left(-T_{c},+T_{c}\right)$ is added.

For simplicity, the simulation is only performed for the power shortage case. In each simulated case, the power shortage is applied by switching on an additional load at $t=$ $2 \mathrm{~s}$. Rate frequency is $60 \mathrm{~Hz}$. Frequency nadir threshold is defined as $59.0 \mathrm{~Hz}$ (for all test cases). Analogous simulation can be conducted for the power surplus case.

\subsection{Simulation results}

For a certain operational time interval, the proposed PFRR is used to reschedule NZI-DGs and battery for improving the frequency dynamic performance. The performance is compared in the following three cases. The status and power of the NZI-DGs in these cases are shown in Table 1. The storage at steady-state for Cases 1-3 are $50,0,0 \mathrm{~kW}$, respectively.

The frequency dynamic performance under the proposed PFRR is shown in Fig. 3. It can be seen that the frequency response can be divided into three phases: the frequency declines before the action of battery (ROCOF limited by inertia), the frequency declines after the power injection of battery (ROCOF is reduced), and frequency reaching nadir point (the actual nadir is successfully maintained above the prescribed threshold by the PFRR). 


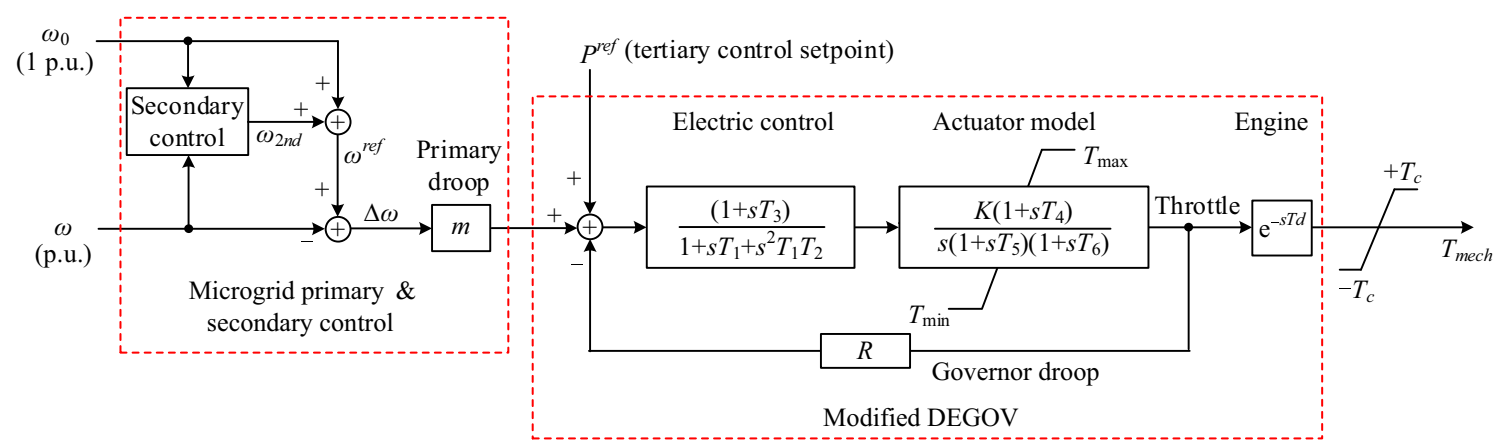

Fig. 2 Modified governor (DEGOV) model integrated with microgrid primary and secondary control functions

Table 1 Control actions and NZI-DG power in Cases 1-3

\begin{tabular}{llll}
\hline DG & Case 1 & Case 2 & Case 3 \\
\hline 1 & OFF & ON, 10 & ON, 10 \\
2 & OFF & ON, 8 & ON, 8 \\
3 & OFF & OFF & ON, 11 \\
4 & OFF & OFF & ON, 10 \\
5 & OFF & OFF & ON, 9 \\
6 & OFF & OFF & OFF \\
7 & OFF & OFF & OFF \\
8 & OFF & OFF & OFF \\
9 & OFF & OFF & OFF \\
10 & OFF & OFF & OFF \\
\hline
\end{tabular}

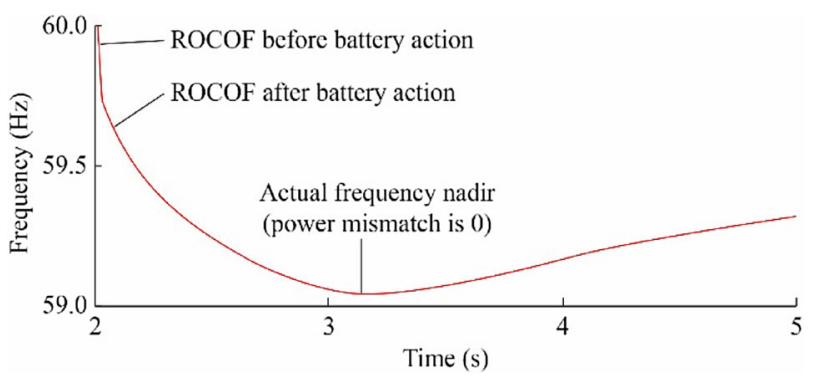

Fig. 3 Frequency response using the proposed PFRR (Case 2)

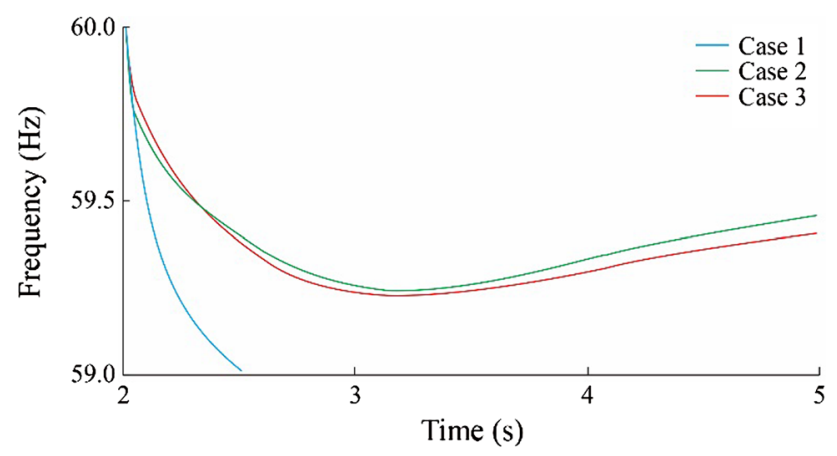

Fig. 4 Comparison of frequency response performances among different cases
The simulation results in the three cases are compared in Table 1 and Fig. 4, which are discussed as follows.

Case 1: it is benchmark (PFRR is not used). The DGs are scheduled by the economic dispatch model (the economic dispatch model is not the focus of this paper). The sum of the maximum power of the ZI-DGs (wind and PV) is $550 \mathrm{~kW}$. Thus the battery is being charged at $50 \mathrm{~kW}$ as shown in Table 1. The microgrid mechanical inertia with all the NZI-DGs off is only $5 \mathrm{~kW} \cdot \mathrm{s} / \mathrm{Hz}$, which is provided by the ZI-DG.

In fact, this is a case that all the online DGs are ZI-DGs. So the microgrid has zero rotational inertia. As shown by the blue curve in Fig. 4, the frequency dropped below the nadir point (collapse) before the time at which the battery power can arrest the frequency. This is because of the very large ROCOF due to the zero-inertia of the system. The power injection of the battery can help arrest the frequency decline, but the frequency drops below the nadir threshold before it is being controlled by the action of battery. This verifies the necessity of implementing the PFRR.

Case 2: the proposed PFRR tool is used to adjust the DG schedule and battery power in preparation for a sudden power shortage/surplus of $150 \mathrm{~kW}$ (applied by a step change of load). The results show that the battery power at steady state is regulated at $0 \mathrm{~kW}$ and two NZI-DGs are turned on, and the total power of NZI-DG is dispatched at $482 \mathrm{~kW}$, which are determined by the solution to the PFRR model. On one hand, each of them has an inertia constant of $3 \mathrm{~s}$ so they increase the mechanical inertia of microgrid from $5 \mathrm{~kW} \cdot \mathrm{s} / \mathrm{Hz}$ to $20 \mathrm{~kW} \cdot \mathrm{s} / \mathrm{Hz}$. On the other hand, they will provide the PFR ramp rate (up and down). Suppose only PFR ramp-up is considered, it could be expected that their output will be equal to their $P_{\min }(0)$ due to the minimization of 15.1 .

The frequency response is shown by the green curve in Fig. 4. It can be seen that the initial frequency drop is arrested by the inertia provided by the two DGs, which is different from Case 1. The battery injects $100 \mathrm{~kW}$ (full capacity) to compensate a portion of the power shortage. This power injection after $t_{B}=2.001 \mathrm{~s}$ (the disturbance 


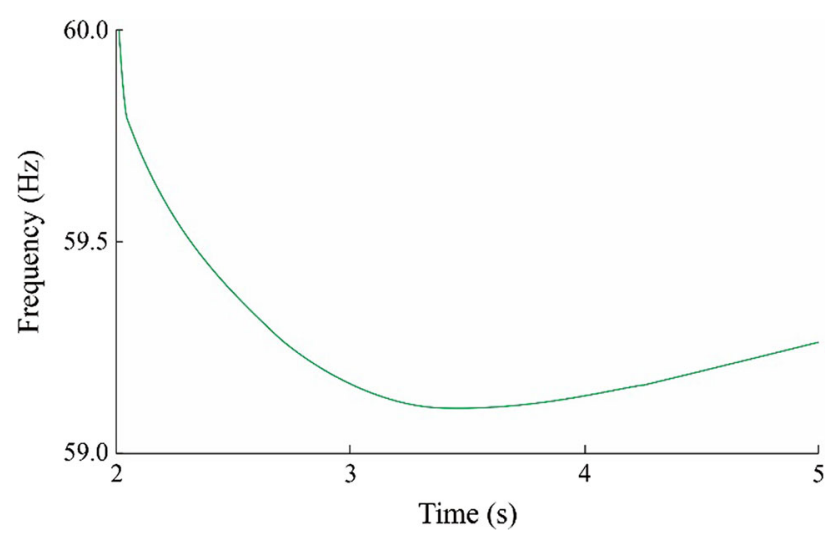

Fig. 5 Frequency response in Case 4

occurs at $t_{0}=2 \mathrm{~s}$ ) should be differentiated from the battery power dispatch shown in Table 1. Once the battery takes action, the ROCOF is approximately $1.67 \mathrm{~Hz} / \mathrm{s}$ and will be reduced as the NZI-DGs providing the primary frequency response. As the mechanical power of NZI-DG ramps up, the gap between the input and output power of the generator becomes smaller. When it becomes zero, the frequency reaches its nadir point. Fig. 4 shows that the actual frequency nadir is successfully kept above the prescribed value $(59.0 \mathrm{~Hz})$. Next, the primary and secondary control of the microgrid starts to restore the frequency.

This verifies that the following processes when the microgrid adjusted by the proposed PFRR experiences a disturbance: the ROCOF is limited by the system inertia; the frequency nadir point is kept above the secured value by the PFR source ramp (governor ramp in micro-turbines). And then the microgrid primary and secondary controls will increase the DG output and restore the frequency back to the rated value.

Case 3: similar to Case 2, the proposed PFRR tool is used in this case to adjust the DG schedule and battery power in preparation for a sudden power shortage/surplus with the same prescribed threshold of frequency nadir. But the power change is $200 \mathrm{~kW}$.

In this case, in preparation for the higher level of power shortage, more NZI-DGs are needed to provide the inertia and the PFR to the microgrid. The system inertia increases to $30 \mathrm{~kW} \cdot \mathrm{s} / \mathrm{Hz}$.

The frequency response is shown by the red curve in Fig. 4. Figure 4 shows the actual ROCOF is $1.667 \mathrm{~Hz} / \mathrm{s}$ after the battery action and the actual frequency nadir is also kept above the prescribed threshold $(59.0 \mathrm{~Hz})$ even though the power shortage is larger than that in Case 2. More NZI-DGs are needed to be online to support the required frequency dynamic performance. This is because the system needs a larger PFR ramp-up capability to increase the mechanical power faster and therefore maintain the frequency nadir point above the threshold even under a larger disturbance.

Case 4: An additional comparative case is presented in Fig. 5, in which the same PFRR as that in Case 2 is applied, but $c_{i}$ of each NZI-DG is reduced to be $15 \mathrm{~kW} / \mathrm{s}$. The results show that, although the initial ROCOF is the same, the DGs provide slower PFR ramp, the frequency and therefore the system will have larger frequency declines and the actual nadir point will be below the threshold $(59.0 \mathrm{~Hz})$.

\section{Conclusion}

In this paper, the PFRR strategy is proposed for enhancing the microgrid resilience in terms of frequency dynamics in case of disturbances in the islanded operation mode. The PFRR can ensure the ROCOF and frequency nadir performance criteria. The relationships among system inertia, ROCOF, frequency nadir, PFR as well as primary/ secondary controls of the microgrid are analyzed and investigated. These measures enhance the microgrid resilience and survivability, which is essential for serving the critical loads in risky events. This work also contributes to further investigation of effective resolution to the low inertia issue of microgrids.

Future research will further investigate the impacts of the ramp rate of the battery and size on the frequency nadir after a sudden power change in the islanded operation mode.

Open Access This article is distributed under the terms of the Creative Commons Attribution 4.0 International License (http:// creativecommons.org/licenses/by/4.0/), which permits unrestricted use, distribution, and reproduction in any medium, provided you give appropriate credit to the original author(s) and the source, provide a link to the Creative Commons license, and indicate if changes were made.

\section{References}

[1] U.S. Department of Energy (2009) Operates resiliently against attack and natural disaster. http://www.smartgrid.gov/files/ operates_resiliently_against_attack_and_natural_disaster_102009.pdf. Accessed 3 March 2018

[2] Che L, Khodayar M, Shahidehpour M (2014) Only connect: microgrids for distribution system restoration. IEEE Power Energy Mag 12(1):70-81

[3] Che L, Shahidehpour M (2019) Adaptive formation of microgrids with mobile emergency resources for critical service restoration in extreme conditions. IEEE Trans Power Syst 34(1):742-753

[4] Wu WH, Chen YD, Luo A et al (2017) A virtual inertia control strategy for DC microgrids analogized with virtual synchronous machines. IEEE Trans Ind Electron 64(7):6005-6016 
[5] Zhong Q, Weiss G (2011) Synchronverters: inverters that mimic synchronous generators. IEEE Trans Ind Electron 58(4):1259-1267

[6] Soni N, Doolla S, Chandorkar MC (2013) Improvement of transient response in microgrids using virtual inertia. IEEE Trans Power Del 28(3):1830-1838

[7] Ketabi A, Fini MH (2015) An under-frequency load shedding scheme for hybrid and multiarea power systems. IEEE Trans Smart Grid 6(1):82-91

[8] Shekari T, Aminifar F, Sanaye-Pasand M (2016) An analytical adaptive load shedding scheme against severe combinational disturbances. IEEE Trans Power Syst 31(5):4135-4143

[9] Karimi M, Wall P, Mokhlis H et al (2017) A new centralized adaptive under-frequency load shedding controller for microgrids based on a distribution state estimator. IEEE Trans Power Del 32(1):370-380

[10] Wang Z, Chen B, Wang J et al (2016) Networked microgrids for self-healing power systems. IEEE Trans Smart Grid 7(1):310-319

[11] Ulbig A, Borsche TS, Andersson G (2014) Impact of low rotational inertia on power system stability and operation. IFAC Proceedings Volumes 47(3):7290-7297

[12] Somuah CB, Schweppe FC (1981) Economic dispatch reserve allocation. IEEE Trans Power App Syst PAS-100(5):2635-2642

[13] Chávez H, Baldick R, Sharma S (2014) Governor rate-constrained OPF for primary frequency control adequacy. IEEE Trans Power Syst 29(3):1473-1480

[14] Wen Y, Li W, Huang G et al (2016) Frequency dynamics constrained unit commitment with battery energy storage. IEEE Trans Power Syst 31(6):5115-5125

[15] Chávez H, Baldick R, Sharma S (2014) Governor rate-constrained OPF for primary frequency control adequacy. IEEE Trans Power Systems 29(3):1473-1480

[16] Chvez H, Baldick R, Matevosyan J (2015) The joint adequacy of AGC and primary frequency response in single balancing authority systems. IEEE Trans Sustain Energy 6(3):959-966

[17] ERCOT nodal operating guides, section 8c: turbine governor speed tests (2015) http:// www.ercot.com/content/wcm/libraries/ 75965/September_25_2015_Nodal_Operating_Guides.pdf. Accessed 3 June 2018

[18] Chavez H, Baldick R, Sharma S (2012) Regulation adequacy analysis under high wind penetration scenarios in ERCOT nodal. IEEE Trans Sustain Energy 3(4):743-750
[19] Sharma S, Huang S, Sarma NDR (2011) System inertial frequency response estimation and impact of renewable resources in ERCOT interconnection. In: Proceedings of IEEE PES general meeting, San Diego, USA, 24-29 July 2011, 6 pp

[20] Sherali HD, Adams WP (1998) A reformulation-linearization technique for solving discrete and continuous nonconvex problems. Springer, Berlin

[21] IEEE test feeder systems (2018) http://sites.ieee.org/pestestfeeders/resources/. Accessed 3 June 2018

[22] Siemens (2018) PSS/E BOSL controllers, DEGOV model. http://www.energy.siemens.com/us/pool/hq/services/powertransmission-distribution/power-technologies-international/ software-solutions/BOSL_Controllers_Standard-1_2008_03_ 13.pdf. Accessed 3 June 2018

Liang CHE received the B.S. degree from Shanghai Jiaotong University, China, in 2006 and the Ph.D. degree from Illinois Institute of Technology, Chicago, USA in 2015, both in Electrical Engineering. He is a professor at Hunan University, China. He was a power system planning consultant at Siemens PTI, Minnetonka, USA in 2015-2016, and an energy management system engineer at the Midcontinent Independent System Operator (MISO), Carmel, USA, in 2017-2019. His research interests include microgrid operation and planning, and power system security analysis.

Xinwei SHEN received the B.S. and Ph.D. degree from the Department of Electrical Engineering, Tsinghua University, China, in 2010 and 2016, respectively. He is now a Post-Doc Fellow in Tsinghua-Berkeley Shenzhen Institute, China. He used to be a Visiting Researcher at Illinois Institute of Technology, USA in 2014 and at U.C. Berkeley, USA in 2017. His current research interests include power system planning, energy internet (integrated energy system) planning and operation.

Mohammad SHAHIDEHPOUR received the Honorary Doctorate degree from the Polytechnic University of Bucharest, Bucharest, Romania. He is a University Distinguished Professor and Bodine Chair Professor and Director of the Robert W. Galvin Center for Electricity Innovation at Illinois Institute of Technology, USA. He is a member of the US National Academy of Engineering and a Fellow of the American Association for the Advancement of Science (AAAS). 